\title{
TRUNCATED STOP LOSS AS OPTIMAL REINSURANCE AGREEMENT IN ONE-PERIOD MODELS
}

\author{
BY
}

\author{
MAREK KALUSZKA
}

\begin{abstract}
We consider several one-period reinsurance models and derive a rule which minimizes the ruin probability of the cedent for a fixed reinsurance risk premium. The premium is calculated according to the economic principle, generalized zero-utility principle, Esscher principle or mean-variance principles. It turns out that a truncated stop loss is an optimal treaty in the class of all reinsurance contracts. The result is also valid for models not involving ruin probability. An example is the Arrow model with the Kahneman-Tversky value function.
\end{abstract}

\section{KEYWORDS}

Reinsurance, insurance, stop loss, truncated stop loss, ruin, optimal contracts, Kahneman-Tversky value function, ruin probability.

\section{INTRODUCTION}

Suppose an insurer decides to buy a reinsurance contract written on the total claim amount basis, i.e. the reinsurer covers $R(X)$ and the cedent covers $X-$ $R(X)$, where $R(X)$ is a compensation function and $X$ is a non-negative random variable on a given probability space $(\Omega, \mathcal{S}, \mathbb{P})$, which represents the total claim size of a portfolio at the end of a fixed time period. The following rules are most common:

- quota share $R(X)=b X, \quad 0<b<1$,

- stop loss $R(X)=(X-a)_{+}, \quad a>0$,

- limited stop loss $R(X)=\min \left((X-a)_{+}, l\right), \quad a, l>0$,

where $a_{+}=a \mathbf{1}(a>0)$. Other types of (re)insurance contracts can be found e.g. in Verlaak and Beirlant (2003). Throughout the paper, we denote by $\mathbf{1}(c)$ the indicator function, i.e. $\mathbf{1}(c)=1$ if the condition $c$ is true and $\mathbf{1}(c)=0$ if it is not. To simplify notation, we write $R=X$ (resp. $R \leq X)$ if the probability that $R \neq X($ resp. $R>X)$ is zero. 
One of the rational approaches to choosing (re)insurance contracts is to minimize the ruin probability of the cedent in a discrete time model (Schäl, 2003, Schäl, 2004) and in a continuous time model (Gaier et al., 2003, Schmidli, 2001, Schmidli, 2002, Taksar and Markussen, 2003, Hipp and Vogt, 2003, Hipp, 2004). The results on controlling the probability of ruin in models without reinsurance can be found in Grandell (1991), Rolski et al. (1999) and Asmussen (2000) among others.

Another approach relies on maximizing dividend payments. See Pechlivanides (1978), Højgaard and Taksar (1999), Asmussen et al. (2000), Højgaard and Taksar (2001), Hipp (2003), and Gerber and Shiu (2004). The third method of constructing optimal contracts is based on maximizing stability measured by the variance or other functionals. See Rantala (1989), Rytgaard (2004), Kaluszka (2004bc) and the papers cited therein. The last (but not least) method relies on maximizing the expected utility of wealth or the return function (Højgaard and Taksar, 1998, Irgens and Paulsen, 2002, Irgens and Paulsen, 2003, Hipp and Plum, 2003).

However a serious limitation of these approaches is that the optimal contracts are derived only in narrow classes of (re)insurance treaties, e.g. quota share or excess-of-loss. What is more, the premium of the reinsurer is mostly calculated via the expected value principle. The aim of the paper is to partly fill this gap. We consider a few one period models and derive a rule which minimizes the probability of the cedent's ruin over the set of all policies for a fixed reinsurer's premium. The premium of the reinsurer is calculated according to the economic principle, generalized zero-utility principle, Esscher principle or mean-variance principles. The ruin probability is neither a concave nor convex functional. Therefore a completely different method from that of Kaluszka (2004a) was applied in the paper. We found that an optimal rule is not a stop loss or quota share but either a truncated stop loss of the form

$$
R(x)=(x-a)_{+} \mathbf{1}(x<b)= \begin{cases}0 & \text { if } x \leq a \\ x-a & \text { if } a<x<b \\ 0 & \text { if } x \geq b,\end{cases}
$$

with $0 \leq a<b<\infty$ or a combination of truncated stop loss with stop loss. A truncated stop loss is also optimal in models not involving ruin probability (see Theorems 8 and 9 of the paper). This contrasts with the approach frequently used in the actuarial literature. The hypothesis is that the rule (1.1) may also be optimal in the class of all rules in discrete time models.

To keep things simple, if not stated otherwise, we assume that $X$ has a distribution function $F(t)=\mathbb{P}(X \leq t)$ of the form

$$
F(t)=p \mathbf{1}(t \geq 0)+(1-p) \int_{0}^{t} f(x) d x \mathbf{1}(t>0)
$$

in which $0 \leq p<1$ and $f$ is a positive density function on $(0, \infty)$. 


\section{Minimizing THE RUIN PROBABILITY}

\subsection{The economic principle}

Let $w_{0}, P$ and $\pi$ denote, respectively, the wealth of the cedent, the reinsurer's premium and the cedent's premium at time 0 . Assume the economic principle, also called the adjusted distribution principle, is applied to calculate the reinsurer's premium, that is, $P=\mathbb{E}(R \phi)$, where $\phi=\phi(X)$ is a non-negative function such that $\mathbb{E} \phi(X)=1$. The economic principle can be derived from expected utility theory as applied to the problem of risk exchanges (see Bühlmann, 1980, 1984, Wang, 2003, Young, 2004). The function $\phi$, called the price density, could be understood as an alteration of the actuarially objective probabilities. The premium $\Pi(R)=\mathbb{E}(R \phi)$ satisfies several desirable axioms like: A1) no unjustified risk loading $\Pi(c)=c, \mathrm{~A} 2)$ no rip-off $\Pi(R) \leq \sup R, \mathrm{~A} 3)$ scale invariance $\Pi(c R)=$ $c \Pi(R)$, A4) translation invariance $\Pi(R+c)=\Pi(R)+c$, where $c$ is a real.

Suppose the cedent wants to have a rule $R$ which minimizes his/her ruin probability at time 1 for a fixed premium of the reinsurer:

$$
\min \mathbb{P}(X-R>w) \text { s.t. } \mathbb{E}(R \phi)=P, L \leq R \leq U,
$$

where $w=w_{0}+\pi-P \geq 0$ and $L=L(X), U=U(X)$ are given constraints such that $0 \leq L \leq U \leq X$.

\section{Theorem 1.}

The rule $R^{*}=R^{*}(X)$ defined by

$$
R^{*}(x)= \begin{cases}x-w & \text { if } L(x) \leq x-w \leq U(x) \text { and } \\ & c(x-w-L(x)) \phi(x)<1, \\ L(x) \quad \text { otherwise, }\end{cases}
$$

is a solution to (2.1) with $c>0$ being such that $\mathbb{E}\left(R^{*} \phi\right)=P$.

Proof. Put $\mathcal{R}=\{R ; \mathbb{E}(R \phi)=P, L \leq R \leq U\}$. It is clear that for any $c>0$

$$
\begin{aligned}
\min _{R \in \mathscr{R}} \mathbb{P} & (X-R>w)= \\
& =\min _{R \in \mathscr{R}} \int[1(x-R(x)>w)+c R(x) \phi(x)] d F(x)-c P \\
& \geq \int_{L(x) \leq y \leq U(x)}[1(x-y>w)+c y \phi(x)] d F(x)-c P \\
& =\int\left[1\left(x-R^{*}(x)>w\right)+c R^{*}(x) \phi(x)\right] d F(x)-c P \\
& =\mathbb{P}\left(X-R^{*}>w\right) .
\end{aligned}
$$

The proof is complete. 
Example 1. Assume the reinsurer's premium is calculated via the expected value principle with safety loading $\beta>0$, which means that $P=(1+\beta) \mathbb{E} R$. The cedent seeks a rule minimizing his/her ruin probability:

$$
\min \mathbb{P}(X-R>w) \text { s.t. } \mathbb{E} R=P_{\beta}, 0 \leq R \leq X,
$$

where $0<P_{\beta}<\mathbb{E} X<\infty$. Hereafter, $P_{\beta}=P /(1+\beta)$. We assume that $P_{\beta}<\mathbb{E}(X-w)_{+}$, otherwise $R(X)=(X-c)_{+}$with $c \leq w$ such that $\mathbb{E} R(X)=P_{\beta}$ is an optimal rule and $\mathbb{P}(X-R(X)>w)=0$. The problem (2.3) was solved by Gajek and Zagrodny (2003) via the Neyman-Pearson lemma (see Gajek and Zagrodny, 2004). We were inspired by a well-known approach from the optimization theory. Theorem 1 implies that a solution to (2.3) is the truncated stop loss:

$$
R_{b^{*}}=(X-w)_{+} \mathbf{1}\left(X<b^{*}\right),
$$

where $b^{*}$ is a positive real such that $\mathbb{E} R_{b^{*}}=P_{\beta}$. If $F$ is continuous on $[w, \infty)$ and if $0<P<\min \left\{w_{0}+\pi,(1+\beta) \mathbb{E}(X-w)_{+}\right\}$, then the existence of $b^{*}$ follows easily from the Darboux property. In fact, by the dominated convergence, $\mathbb{E} R_{b} \rightarrow$ $\mathbb{E}(X-w)_{+}$as $b \rightarrow \infty$ and $\mathbb{E} R_{b} \rightarrow 0$ as $b \rightarrow w$. From the Fubini theorem, we get

$$
\begin{gathered}
\mathbb{E} R_{b}=\int_{w}^{b} \int_{w}^{b} \mathbf{1}(t<x) d t d F(x)=\int_{w}^{b} \int_{t}^{b} d F(x) d t \\
=(b-w) F(b)-\int_{w}^{b} F(t) d t,
\end{gathered}
$$

so the function $b \rightarrow \mathbb{E} R_{b}$ is continuous on $[w, \infty)$. Observe that if $X$ has an atom at $x_{0}>w$, then the function $b \rightarrow \mathbb{E} R_{b}$ is not continuous at $x_{0}$. In consequence, there is no $b$ such that $(1+\beta) \mathbb{E} R_{b}=P$ for some $P$ and we have to change the reinsurer's premium.

For concreteness, let $X$ have the Pareto distribution with parameters 2, $a$, i.e. $F(x)=\left(1-a^{2}(a+x)^{-2}\right) \mathbf{1}(x \geq 0)$, where $a>0$. Let $0<P_{\beta}<a^{2} /(a+w)$ and let $P<w_{0}+\pi$. A solution to (2.3) is the rule

$$
R^{*}=(X-w)_{+} 1\left(X<\frac{a A+1+\sqrt{(a+w) A+1}}{-A}\right)
$$

with $A=P_{\beta} a^{-2}-(a+w)^{-1}$. The ruin probability equals

$$
\mathbb{P}\left(X-R^{*}>w\right)=\frac{(a A)^{2}}{(1+\sqrt{1+(a+w) A})^{2}} .
$$

\subsection{The generalized zero-utility principle}

Suppose the cedent can buy a payment of $R$ for a premium of $P$ such that $\mathbb{E} v(R, P)=0$, where an increasing and continuous function $x \rightarrow v(x, P)$ defines the premium principle. Examples include the 
- mean-value principle $P=u^{-1}(\mathbb{E} u(R))$, where $u$ is increasing and continuous,

- zero-utility principle $\mathbb{E} u(P-R)=u(0)$ with a utility function $u$,

- Swiss premium principle $\mathbb{E} u(R-z P)=u((1-z) P)$ for some $z \in[0,1]$ and some increasing function $u$,

- Orlicz premium principle $\mathbb{E} \psi(R / P)=1$, where $\psi$ is a Young function,

- net premium principle with cost $c P=\mathbb{E} R+\mathbb{E} c(R), c$ being a nondecreasing and continuous function on $[0, \infty)$.

Gerber (1979), Goovaerts et al. (1984), and Goovaerts et al. (2003) studied these principles in detail. Our problem is to determine the rule which minimizes the ruin probability subject to a fixed reinsurance premium $P$ :

$$
\min \mathbb{P}(X-R>\text { w) s.t. } \mathbb{E} v(R, P)=0,0 \leq R \leq X .
$$

Recall that $w$ stands for the wealth of the cedent at time 0 .

\section{Theorem 2.}

If $v(0, P)<0<\mathbb{E} v\left((X-w)_{+}, P\right)<\infty$, then a solution to the problem (2.6) is the truncated stop loss $R_{b^{*}}=(X-w)_{+} \mathbf{1}\left(X<b^{*}\right)$, where $b^{*}$ is a real such that $\mathbb{E} v\left(R_{b^{*}}, P\right)=0$.

Proof. The proof is quite similar to that of Theorem 1. The existence of $b^{*}$ follows from the continuity of the function $b \rightarrow \mathbb{E} v\left(R_{b}, P\right)$, where $R_{b}=(X-w)_{+} \mathbf{1}(X<b)$. Indeed, $v(0, P) \leq v\left(R_{b}, P\right) \leq v\left((X-w)_{+}, P\right)$. By the dominated convergence and (1.2),

$$
\lim _{t \rightarrow b} \mathbb{E} v\left(R_{t}, P\right)=\mathbb{E} \lim _{t \rightarrow b} v\left(R_{t}, P\right)=\mathbb{E} v\left(R_{b}, P\right)
$$

for all $b$, as desired.

Example 2. Assume the reinsurer's premium is calculated according to the zero-utility principle with a utility function, $u$, such that $u^{\prime}>0$. Suppose that $\mathbb{E} u\left(P-(X-w)_{+}\right)<u(0)$, where $P>0$. From Theorem 2 it follows that the ruin probability of the cedent is minimized by the rule $R_{b^{*}}=(X-w)_{+} \mathbf{1}\left(X-b^{*}\right)$, where $b^{*}$ is such that $\mathbb{E} u\left(P-R_{b^{*}}\right)=u(0)$.

\subsection{The Esscher principle}

The mapping $R \rightarrow \mathbb{E}\left(R e^{a R}\right) / \mathbb{E} e^{a R}$ is known as the Esscher premium with parameter $a>0$. The Esscher premium principle was devised by Bühlmann (1980). For more information about this principle we refer the reader to Bühlmann (1980), Goovaerts et al. (1984), Gómez-Déniz et al. (1999) and Embrechts (2000). The criterion for selecting the optimal rule is the minimization of the ruin probability provided a fixed reinsurer's premium is calculated by the Esscher principle:

$$
\min \mathbb{P}(X-R>w) \text { s.t. } P=\operatorname{Ess}_{a}(R), 0 \leq R \leq X,
$$

where $\operatorname{Ess}_{a}(R)=\mathbb{E}\left(R e^{a R}\right) / \mathbb{E} e^{a R}$. 


\section{Theorem 3.}

Assume $0<P \leq a^{-1}$ and assume $0<\mathbb{E}\left[\left((X-w)_{+}-P\right) \exp \left(a(X-w)_{+}\right)\right]<\infty$. A solution to (2.7) is the truncated stop loss rule $R_{b^{*}}=(X-w)_{+} \mathbf{1}\left(X<b^{*}\right)$ with $b^{*}$ being such that $P=\operatorname{Ess}_{a}\left(R_{b^{*}}\right)$.

Proof. Observe that $P=\operatorname{Ess}_{a}(R)$ iff $\mathbb{E}[(R-P) \exp (a R)]=0$. Thus, for any $c>0$

$$
\begin{aligned}
& \min _{R} \mathbb{P}(X-R>w)= \\
& =\min _{R} \int[\mathbf{1}(x-R(x)>w)+c(R(x)-P) \exp (a R(x))] d F(x) .
\end{aligned}
$$

The rest of the proof is similar to that of Theorem 1. Put $\psi(b)=\mathbb{E}\left[\left(R_{b}-P\right)\right.$ $\left.\exp \left(a R_{b}\right)\right]$ with $R_{b}=(X-w)_{+} \mathbf{1}(X<b)$. Clearly, $\psi(w)=-P<0, \psi(\infty)>0$, and $\psi$ is continuous. Therefore, there exists $b^{*}$ such that $\psi\left(b^{*}\right)=0$.

Remark 1. Unless the assumptions of Theorem 3 are satisfied, other rules may be optimal. For instance, if $\max \left(a^{-1}, \operatorname{Ess}_{a}\left(\hat{R}_{0}\right)\right)<P<E s s_{a}\left(\hat{R}_{\infty}\right)$ with $\hat{R}_{b}=\min$ $\left(X, P-a^{-1}\right)+\left(X-w-P+a^{-1}\right)_{+} \mathbf{1}(X<b)$, then there is $b^{*}$ such that $\operatorname{Ess}_{a} \hat{R}_{b^{*}}=P$ and the rule $\hat{R}_{b^{*}}$ solves (2.7).

\subsection{Mean-variance principles}

Suppose the cedent wants to minimize the ruin probability for a fixed reinsurer's premium, say $P$, based on the mean and the variance of the compensated part of the risk. Suppose also that the cedent wants to control his/her expected gain and is willing to pay not more than $P_{0}$ for reinsurance. As a consequence, the following problem arises

$$
\min \mathbb{P}(X-R>w) \text { s.t. } R \in \mathcal{R}(M)
$$

where $\mathcal{R}(M)=\left\{R ; R=R(X), 0 \leq R \leq X, \mathbb{E} R \leq f\left(P_{0}, \mathbb{D} R\right), \mathbb{E} R \leq M\right\}$, and $t \rightarrow$ $f(P, t)$ is a decreasing function defining a premium principle. Examples of reinsurer's premium principles include the

- standard deviation principle $P=\mathbb{E} R+\beta \mathbb{D} R ; f=P-\beta$,

- variance principle $P=\mathbb{E} R+\beta \mathbb{D}^{2} R ; f=P-\beta t^{2}$,

- mixed principle $P=\mathbb{E} R+\alpha \mathbb{D} R+\beta \mathbb{D}^{2} R ; f=P-\alpha t-\beta t^{2}$,

- modified variance principle $P=\mathbb{E} R+\beta \mathbb{D}^{2} R / \mathbb{E} R ; f=\frac{P+\sqrt{P^{2}-4 \beta t^{2}}}{2}$,

- mean value principle $P=\left[\mathbb{E}\left(R^{2}\right)\right]^{1 / 2}=\left[(\mathbb{E} R)^{2}+\mathbb{D}^{2} R\right]^{1 / 2} ; f=\sqrt{P^{2}-t^{2}}$,

- quadratic utility principle $P=\mathbb{E} R+c-\left(c^{2}-\mathbb{D}^{2} R\right)^{1 / 2} ; f=P-c+\sqrt{c^{2}-t^{2}}$,

where $\alpha, \beta, c>0, \mathbb{D} R$ means the standard deviation of random variable $R$ and $\mathbb{D}^{2} R$ denotes the variance. Let $b(m)$ be the solution to $\int_{w}^{b}(x-w) d F(x)=m$ in $b>w$. 


\section{Theorem 4.}

Suppose $0<M<\mathbb{E}(X-w)_{+}$. The truncated stop loss $R_{M}=(X-w)_{+} \mathbf{1}(X<b(M))$ is a solution to (2.8) provided $\mathbb{E} R_{M} \leq f\left(P_{0}, \mathbb{D} R_{M}\right)$.

Proof. Put $\mathcal{R}_{m}=\{R ; 0 \leq R \leq X, \mathbb{E} R=m\}$ and put $R_{m}=(X-w)_{+} \mathbf{1}(X<b(m))$. It is clear that $R_{m} \in R_{m}$. Given $c>0$, we have

$$
\begin{gathered}
\min _{R \in \mathcal{R}(M)} \mathbb{P}(X-R>w) \geq \min _{0 \leq m \leq M} \min _{R \in \mathcal{R}_{m}} \mathbb{E}[\mathbf{1}(X-R>w)+c(R-m)] \\
\geq \min _{0 \leq m \leq M} \mathbb{E} \min _{R \in \mathcal{R}_{m}}[\mathbf{1}(X-R>w)+c(R-m)]=\min _{0 \leq m \leq M} \mathbb{P}\left(X-R_{m}>w\right) \\
=\min _{0 \leq m \leq M} \mathbb{P}(X \geq b(m))=\mathbb{P}(X \geq b(M))=\mathbb{P}\left(X-R_{M}>w\right) .
\end{gathered}
$$

By the assumptions, $R_{m} \in R_{m}$, which completes the proof.

Example 3. Suppose the standard deviation principle is applied to calculate the reinsurer's premium and suppose the cedent is willing to minimize the ruin probability. A solution to the problem

$$
\min \mathbb{P}\left(X-R>\text { w) s.t. } 0 \leq R \leq X, \mathbb{E} R+\beta \mathbb{D} R \leq P_{0}, \mathbb{E} R \leq M,\right.
$$

is the truncated stop loss $R=(X-w)_{+} \mathbf{1}(X<b)$, where $b$ is such that $\mathbb{E} R=M$ and $\mathbb{E} R+\beta \mathbb{D} R \leq P_{0}$.

Remark 2. A similar result can be formulated for the semi-variance principle of the form $P=\mathbb{E} R+g\left(\mathbb{D}^{+} R\right)$, where $\left(\mathbb{D}^{+} R\right)^{2}=\mathbb{E}\left[(R-\mathbb{E} R)_{+}\right]^{2}$ is the semi-variance and $g$ is an increasing and continuous function on $[0, \infty)$.

\subsection{Reinsurance and investment}

Let $w \geq 0$ denote the cedent's wealth at time 0 after paying the premium $P$ for reinsurance. At time 0 , the cedent invests a part of his/her wealth, say $a w$, into risky assets with a rate of return, $r$, being a random variable independent of $X$, with a distribution function $G$ and the continuous density $g=G^{\prime}$. At time 1, the wealth of the cedent is equal to

$$
(1-a) w+(1+r) a w-X+R=(1+a r) w-X+R .
$$

For the premium calculation the reinsurer uses the expected value principle with safety loading $\beta>0$. The cedent not only seeks for a reinsurance rule minimizing ruin probability, but also wants to known how much of wealth he should invest into risky assets. Short sale is rejected, so the following constrained optimization problem arises

$$
\min \mathbb{P}((1+a r) w-X+R<0) \text { s.t. }(a, R) \in[0,1] \times \mathcal{R}
$$

in which $R=\left\{R ; R=R(X), \mathbb{E} R=P_{\beta}, 0 \leq R \leq X\right\}$. To solve the problem (2.10), we make the following assumption: 
A1. The density $g$ is increasing on $\left(-1, r_{0}\right]$ and is decreasing on $\left[r_{0}, \infty\right)$, where $-1<r_{0}<\infty$.

Let $g^{-1}$ denote the inverse function to $g$ on $\left(-1, r_{0}\right]$. Define $x(a, c)$ to be the solution to the equation

$$
G\left(\frac{x-w}{a w}\right)=G\left(g^{-1}(a c w)\right)+c\left(x-w-a w g^{-1}(a c w)\right)
$$

in $x>w+a w g^{-1}(a c w)$ with $a \in[0,1], c>0$, and $a c w<g\left(r_{0}\right)$. Here and subsequently, we set $a / 0=\infty$ for $a>0$ and $a / 0=-\infty$ for $a \leq 0$. Let $c(a)$ be the solution to $\psi(a, c)=P_{\beta}$, where

$$
\psi(a, c)=\mathbb{E}\left[\left(X-w-a w g^{-1}(a c w)\right)_{+} \mathbf{1}(X<x(a, c))\right]
$$

for $0<c<(a w)^{-1} g\left(r_{0}\right), 0 \leq a \leq 1$.

\section{Theorem 5.}

Suppose $0<P_{\beta}<\mathbb{E}(X-w)_{+}$. Under assumption Al, a solution to (2.10) is the truncated stop loss policy $R_{a^{*}}$, where

$$
R_{a}=\left(X-w-a w g^{-1}(a c(a) w)\right)_{+} \mathbf{1}(X<x(a, c(a)))
$$

and $a^{*}$ is an optimal investment strategy being a minimizer of $a \rightarrow \mathbb{P}((1+a r) w-$ $\left.X+R_{a}<0\right)$ over $[0,1]$.

Proof. Since $X$ and $r$ are independent random variables, we have

$$
\begin{aligned}
\min _{(a, R)} \mathbb{P} & ((1+a r) w-X+R<0)= \\
= & \min _{(a, R)} \int\left[\mathbb{P}\left(a r<\frac{x-R(x)-w}{w}\right)+c R(x)\right] d F(x)-c P_{\beta} \\
& \geq \min _{0 \leq a \leq 1} \int \min _{0 \leq y \leq x} h(y) d F(x)-c P_{\beta},
\end{aligned}
$$

where $0<c<g\left(r_{0}\right) /(a w)$ and $h(y)=G\left(\frac{x-y-w}{a w}\right)+c y$. By a straightforward but lengthy computation, we obtain that the minimum of $h(y)$ is attained at

$$
y(x ; a, c)=\left(x-w-a w g^{-1}(a c w)\right)_{+} \mathbf{1}(x<x(a, c)),
$$

where $x(a, c)$ is defined at (2.11) (compare the value of the local minimum of $h(y)$ with $h(0))$. The solution $x(a, c)$ to (2.11) exists and is unique since $G$ is convex on $\left(-1, r_{0}\right]$, concave on $\left[r_{0}, \infty\right)$, and $x \rightarrow G\left(g^{-1}(a c w)\right)+c\left(x-w-a w g^{-1}(a c w)\right)$ is the tangent line of $G$ at $g^{-1}(a c w)<r_{0}$. Moreover, the function $x(a, c)$ is continuous and $c \rightarrow x(a, c)$ is decreasing for each $a$. The function $c(a)$ is well-defined if $0<P_{\beta}<\mathbb{E}(X-w)_{+}$. Indeed, by the dominated convergence, $\psi(a, c) \rightarrow 0$ as $c \rightarrow$ $g\left(r_{0}\right) /(a w)$ and

$$
\lim _{c \rightarrow 0+} \psi(a, c)=\mathbb{E}(X-w(1-a))_{+} \geq \mathbb{E}(X-w)_{+}>P_{\beta},
$$


where $\psi$ is defined in (2.12). By (1.2), the continuity of $x(a, c)$ and the dominated convergence, $\psi$ is continuous. Moreover, $c \rightarrow \psi(a, c)$ is decreasing. Indeed,

$$
\begin{gathered}
\frac{\partial}{\partial c} \psi(a, c)=a w\left[F \left(w+a w g^{-1}(a c w)-F(x(a, c)] \frac{\partial}{\partial c} g^{-1}(a c w)+\right.\right. \\
+\left[x(a, c)-w-a w g^{-1}(a c w)\right] f(x(a, c)) \frac{\partial}{\partial c} x(a, c)<0
\end{gathered}
$$

because $\frac{\partial}{\partial c} g^{-1}(a w c) \geq 0, \frac{\partial}{\partial c} x(a, c)<0$, and $x(a, c)>w+a w g^{-1}(a c w)$ (see (2.5)). This implies that $c(a)$ exists and the function $a \rightarrow c(a)$ is continuous.

Since $\mathbb{E} y(X ; a, c(a))=\mathbb{E} R_{a}=P_{\beta}$, we have

$$
\min _{(a, R)} \mathbb{P}((1+a r) w-X+R<0) \geq \min _{0 \leq a \leq 1} \mathbb{P}\left((1+a r) w-X+R_{a}<0\right) .
$$

By the dominated convergence and the continuity of $x(a, c)$ and $c(a)$, the function $a \rightarrow \mathbb{P}\left((1+a r) w-X+R_{a}<0\right)$ is continuous on [0,1]. Consequently, $h$ attains its minimum which completes the proof.

Remark 3. Suppose the random variable $1+r$ has a lognormal distribution, i.e. the distribution of $\ln (1+r)$ is normal $N\left(\mu, \sigma^{2}\right)$. Then $G$ satisfies assumption A1 with $r_{0}=\exp \left(\mu-\sigma^{2}\right)-1$.

\section{OTHER MODELS}

\subsection{Minimization of the variance of the cedent's cover}

The problem of minimizing the variance of the cedent's cover was treated in many papers (see e.g. Kaluszka, 2004ab, for a review of literature). Herein we assume that the probability that the reinsurer's cover will be greater than a given level, say $P$, is fixed and equal to $\alpha$. Under this constraint, we will study the problem that consists in choosing a contract which minimizes the variance of the cedent's cover:

$$
\min \mathbb{D}^{2}(X-R) \text { s.t. } \mathbb{P}(R>P)=\alpha, 0 \leq R \leq X,
$$

where $0 \leq \alpha<1$. Observe that $P$ can also be interpreted as a reinsurer's premium calculated according to the percentile principle (see Gerber, 1979, p. 69).

\section{Theorem 6.}

Suppose the distribution of $X$ is given by (1.2) and suppose $0<P \leq F^{-1}(1-\alpha)-$ $\mathbb{E} X$, where $F^{-1}(1-\alpha)$ means the $(1-\alpha)$-quantile of $X$. Then a solution to (3.1) is as follows:

$$
R_{b^{*}}=\left(X-b^{*}\right)_{+}-\left(X-b^{*}-P\right)_{+} \mathbf{1}\left(X<F^{-1}(1-\alpha)\right)
$$

with $b^{*}$ being a minimizer of $\mathbb{E}\left(X-R_{b}-b\right)^{2}$ over $0 \leq b \leq \mathbb{E} X$. 
Proof. Since $\mathbb{D}^{2}(X-R)=\min _{0 \leq b \leq \mathbb{E} X} \mathbb{E}(X-R-b)^{2}$, it is enough to solve the problem

$$
\min \mathbb{E}(X-R-b)^{2} \text { s.t. } \mathbb{P}(R>P)=\alpha, 0 \leq R \leq X .
$$

Set $\mathcal{R}=\{R ; \mathbb{P}(R \geq P)=\alpha, 0 \leq R \leq X\}$. As in the proof of Theorem 1, we obtain

$$
\min _{R \in \mathcal{R}} \mathbb{E}(X-R-b)^{2} \geq \int \min _{0 \leq y \leq x}\left((x-y-b)^{2}+c \mathbf{1}(y>P)\right) d F(x)-c \alpha,
$$

where $c>0$. It is easy to see that the minimium over $y$ is attained at $y_{b c}(x)$, where

$$
y_{b c}(x)= \begin{cases}0 & \text { if } x<b \\ P & \text { if } b+P<x<b+P+\sqrt{c} \\ x-b & \text { otherwise, }\end{cases}
$$

Observe that $F^{-1}(1-\alpha)-b-P \geq F^{-1}(1-\alpha)-\mathbb{E} X-P>0$. Since $\mathbb{P}\left(y_{b c_{0}}(X)>P\right)=\alpha$ for $c_{0}=\left(F^{-1}(1-\alpha)-b-P\right)^{2}$, we have $y_{b c_{0}}(X) \in \mathcal{R}$ for all $b$. The proof is complete.

\subsection{Maximizing utility}

In this section, the optimality criterion that we use is that the cedent's expected utility should be minimized subject to constraints on the reinsurer's expected net profit and the probability that the reinsurance payment will be greater than a level $L$ :

$$
\max \mathbb{E} u(w-X+R) \text { s.t. } P_{\beta}=\mathbb{E} R, \mathbb{P}(R>L)=\alpha, 0 \leq R \leq X,
$$

where $0 \leq \alpha<1$ and $u$ is a von Neumann-Morgernstern utility function of the risk-averse cedent, that is $u^{\prime}(x)>0$ and $u^{\prime \prime}(x)<0$ for all $x$. Recall that $P_{\beta}=$ $P /(1+\beta)$. The problem is a modification of the time-honoured Arrow's problem (1963). Similar modifications have been suggested by Bühlmann in a dynamic setting (see Gaier et al., 2003, and Hipp, 2003).

\section{Theorem 7.}

Assume $X$ has the distribution function (1.2), assume $w+L<F^{-1}(1-\alpha)$ and assume $\mathbb{E} R_{w}<P_{\beta} \leq \mathbb{E} R_{0}$, where $R_{b}=(X-b)_{+}-(X-b-L)_{+} \mathbf{1}\left(X<F^{-1}(1-\alpha)\right)$. Then a solution to (3.3) is the rule $R_{b^{*}}$ in which $b^{*}$ is such that $\mathbb{E} R_{b^{*}}=P_{\beta}$.

Proof. Define $\psi(b)=\mathbb{E} R_{b}-P_{\beta}$. By the dominated convergence, the function $\psi$ is continuous. Since $\psi(0) \geq 0$ and $\psi(w)<0$, there exists $b^{*}$ such that $\psi\left(b^{*}\right)=0$. Put $\mathcal{R}=\left\{R ; \mathbb{E} R=P_{\beta}, \mathbb{P}(R>L)=\alpha, 0 \leq R \leq X\right\}$. Clearly

$$
\begin{aligned}
& \max _{R \leq R} \mathbb{E} u(w-X+R) \leq \\
& =\int \max _{0 \leq y \leq x}\left(u(w-x+y)-c_{1} y-c_{2} \mathbf{1}(y>L)\right) d F(x)+c_{1} P_{\beta}+c_{2} \alpha,
\end{aligned}
$$


where $0<c_{1}<u^{\prime}(w)$ and $c_{2}>0$. The rest of the proof is analogous to that of Theorem 1 .

Example 4. Suppose the reinsurer is willing to have a contract which obliges him to cover $0 \leq R \leq \min (X, L)$, where $L$ is a positive real. From Theorem 7 it follows that a solution to the problem

$$
\max \mathbb{E} u(w-X+R) \text { s.t. } P_{\beta}=\mathbb{E} R, 0 \leq R \leq \min (X, L),
$$

is the limited stop loss of the form

$$
R=(X-b)_{+}-(X-b-L)_{+}=\min (X-b, L),
$$

with $b$ being such that $\mathbb{E} R=P_{\beta}$. This fact is well known.

\subsection{Maximization of the dividend payment}

Assume the expected value principle is used for the calculation of the reinsurance premium. The dividends are paid at time 1 if the gain of the cedent after reinsurance exceeds a given level, say $c$, where $c$ may stand for the expenses plus profit. Suppose the cedent's objective is to maximize the expected dividend payment $\mathbb{E}(\pi-P-X+R-c)_{+}$for a fixed premium of the reinsurer:

$$
\max \mathbb{E}(\pi-P-X+R-c)_{+} \text {s.t. } P_{\beta}=\mathbb{E} R, 0 \leq R \leq X .
$$

\section{Theorem 8.}

If $\mathbb{E}[X \mathbf{1}(X<\pi-P-c)]<P_{\beta}<\mathbb{E} X$, then a solution to (3.5) is given by $R_{b^{*}}=X \mathbf{1}(X<$ $\left.b^{*}\right)$ in which $b^{*}>0$ is a real such that $\mathbb{E} R_{b^{*}}=P_{\beta}$.

Proof. The proof follows along the same lines as in Theorem 1.

It is easy to check that there is a real $P$ satisfying the assumption of Theorem 8 iff $(1+\beta) \mathbb{E}[X \mathbf{1}(X<\pi-c-\mathbb{E} X)]<\mathbb{E} X$.

\subsection{The Kahneman-Tversky value function}

The prospect theory of Kahneman and Tversky (1979) represents an alternative theory of decision making under uncertainty, as opposed to the standard Morgenstern-von Neumann utility theory. Based on a series of experimental observations, Kahneman and Tversky propose a value function defined on the gains or losses relative to a reference point, instead of the absolute level of consumption or wealth. Specifically, they state that the value functions are (i) defined on deviations from the reference point; (ii) generally concave for gains and commonly convex for losses; (iii) steeper for losses than gains. Prospect theory has been widely supported by experimental studies of human behavior. We find a solution to the Arrow problem with the expected Kahneman-Tversky value function as an objective function: 


$$
\max \mathbb{E} u(w-X+R) \text { s.t. } P_{\beta}=\mathbb{E} R, 0 \leq R \leq X,
$$

where $w$ is a reference point and $u$ is an increasing and nondifferentiable at 0 function such that $u^{+}(0)<u^{-}(0), u^{\prime \prime}(x)>0$ for $x<0$, and $u^{\prime \prime}(x)<0$ for $x>0$. Hereafter, $u^{-}(0)$ and $u^{+}(0)$ means the left derivative and the right derivative of $u$ at 0 , respectively.

\section{Theorem 9.}

Assume $0<P_{\beta}<\mathbb{E}\left[(X-w)_{+} \mathbf{1}\left(X<b_{0}\right)\right]$, where $b_{0}$ solves the equation $u(w-b)=$ $u(0)+u^{+}(0)(w-b)$ in $b>w \geq 0$. The truncated stop loss rule $R_{b^{*}}=(X-w)_{+} \mathbf{1}(X<$ $\left.b^{*}\right)$ with $b^{*}$ such that $\mathbb{E} R_{b^{*}}=P_{\beta}$ is a solution to (3.6).

Proof. For every $u^{+}(0)<c<u^{-}(0)$, we have

$$
\max _{R \in \mathcal{R}} \mathbb{E} u(w-X+R) \leq \int \max _{0 \leq y \leq x}(u(w-x+y)-c y) d F(x)+c P_{\beta}
$$

in which $R=\left\{R ; \mathbb{E} R=P_{\beta}, 0 \leq R \leq X\right\}$. The maximum in $y$ is attained at $y_{c}(x)=$ $(x-w)_{+} \mathbf{1}\left(x<x_{c}\right)$, where $x_{c}$ is a solution to $u(w-x)=u(0)+c(w-x)$ in $x>w$. Observe that $w<x_{c}<b_{0}$, which implies $0 \leq y_{c}(x) \leq(x-w)_{+} \mathbf{1}\left(x<b_{0}\right)$ for all $x$. Moreover, $w \leq b \rightarrow \mathbb{E}\left[(X-w)_{+} \mathbf{1}(X<b)\right]$ is a continuous function. As a result, there exists $b^{*}$ such that $\mathbb{E} R_{b^{*}}=P_{\beta}$.

\section{REFERENCES}

Arrow, K.J. (1963) Uncertainty and the welfare economics of medical care. American Economic Review, 53, 941-973.

Asmussen, S., HøjgaArd B. and Taksar, M. (2000) Optimal risk control and dividend distribution policies. Example of excess-of-loss reinsurance for an insurance corporation. Finance and Stochastics, 4, 299-324.

Asmussen, S. (2000) Ruin probabilities. World Scientific, Singapore.

BüHLManN, H. (1980) An economic principle. ASTIN Bulletin, 11, 52-60.

Bühlmann, H. (1984) The general economic premium principle. ASTIN Bulletin, 14, 13-21.

Embrechts, P. (2000) Actuarial versus financial pricing of insurance. Risk Finance, 1, 17-26.

Gómez-DÉniz, E., Hernández-Bastida, A. and VÁzquez-Polo F.J. (1999) The Esscher premium principle in risk theory: a Bayesian sensitivity study. Insurance: Mathematics and Economics, 25, 387-395.

Gaier, J., Grandits, P. and Schachermayer, W. (2003) Asymptotic ruin probabilities and optimal investment. Ann. Appl. Probab., 13, 1054-1076.

GAJeK, L. and ZaGrodny, D. (2003) Oral communication.

GAJEK, L. and ZAGRODNY, D. (2004) Reinsurance arrangements maximizing insurer's survival probability. Journal of Risk and Insurance, 71, 421-435.

Gerber, H.U. (1979) An Introduction to Mathematical Risk Theory. S.S. Huebner Foundation for Insurance Education, Wharton School, University of Pennsylvania, Philadelphia.

Gerber, H.U. and Shiu, E.S.W. (2004) Optimal dividends: analysis with brownian motion. North American Actuarial Journal, 8, 1-20.

Goovaerts, M.J., De Vylder, F. and Haezendonck, J. (1984) Insurance Premiums. NorthHolland Publishing Co., Amsterdam.

Goovaerts, M.J., KaAs, R., Dhaene, J., and TAng, Q. (2003) A unified approach to generate risk measures. Working paper, University of Leuven.

Grandell, J. (1991) Aspects of Risk Theory. Springer-Verlag, New York. 
HIPP, Ch. (2004) Stochastic Control with Application in Insurance. Lecture Notes in Mathematics, Vol. 1856, Springer-Verlag.

Hipp, Ch. and Plum, M. (2003) Optimal investment for insurers with state dependent income, and for insurers. Finance and Stochastics, 7, 299-321.

Hipp, Ch. (2003) Optimal dividend payment under a ruin constraint: discrete time and state space. (http://www.astin2003.de/img/papers/hipp.pdf)

Hipp, Ch. and Vogt, M. (2003) Optimal dynamic XL reinsurance. ASTIN Bulletin, 33, 193-207.

HøjgaARD B. and TAKSAR, M. (1998) Optimal proportional reinsurance policies for diffusion models. Scandinavian Actuarial Journal, 1, 166-168.

HøJgAARD B. and TAKSAR, M. (1999) Controlling risk exposure and dividends payout schemes: insurance company example. Mathematical Finance, 9, 153-182.

HøJGAARD B. and TAKSAR, M. (2001) Optimal risk control for a large corporation in the presence of returns on investments. Finance and Stochastics, 5, 527-547.

IRgens, Ch. and Paulsen, J. (2004) Optimal control of risk exposure, reinsurance and investments for insurance portfolios. Insurance: Mathematics and Economics, 35, 21-51.

Irgens, Ch. and Paulsen, J. (2003) Maximizing terminal utility by controlling risk exposure; a discrete-time dynamic control approach. (http://www.mi.uib.no/ jostein/wpaper.html)

Kahneman, D. and Tversky, A. (1979) Prospect Theory: An analysis of decision under risk. Econometrica, 47, 263-291.

KaluszKa, M. (2004a) An extension of the Gerber-Bühlmann-Jewell conditions for optimal risk sharing. ASTIN Bulletin, 34, 27-48.

KAluszKa, M. (2004b) Mean-variance optimal reinsurance arrangements. Scandinavian Actuarial Journal, 1, 28-41.

Kaluszka, M. (2004c) An extension of Arrow's result on optimality of a stop loss contract. Insurance: Mathematics and Economics, 35, 527-536.

PeChlivanides, P.M. (1978) Optimal reinsurance and dividend payment strategies. ASTIN Bulletin, 10, 34-46.

RANTALA, J. (1989) On experience rating and optimal reinsurance. ASTIN Bulletin, 19, 153-178.

Rolski, T., Schmidli, H., Schmidt, V. and Teugels, J. (1999) Stochastic Processes for Insurance and Finance. John Wiley \& Sons, Chichester.

RytgaArd, M.M. (2004) Stop loss reinsurance. Encyclopedia of Actuarial Science, John Wiley \& Sons, Chichester.

SCHÄL, M. (2003) Stochastic optimization for the ruin probability. Proc. Appl. Math. Mech., 3, 17-19.

SCHÄL, M. (2004) On discrete-time dynamic programming in insurance: exponential utility and minimizing the ruin probability. Scandinavian Actuarial Journal, 3, 189-210.

SchmidLi, H. (2001) Optimal proportional reinsurance policies in a dynamic setting. Scandinavian Actuarial Journal, 2, 55-68.

SchmidLI, H. (2002) On minimizing the ruin probability by investment and reinsurance. Ann. Appl. Probab., 12, 890-907.

TAKSAR, M.I. and MARKuSSEN, Ch. (2003) Optimal dynamic reinsurance policies for large insurance portfolios. Finance and Stochastics, 7, 97-121.

VerlaAK, R. and BeIRlant, J. (2003) Optimal reinsurance programs: an optimal combination of several reinsurance protections on a heterogeneous insurance portfolio. Insurance: Mathematics and Economics, 33, 381-403.

WANG S.S. (2003) Equilibrium pricing transforms: new results using Bühlmann's 1980 economic model. ASTIN Bulletin, 33, 57-73.

Young, V.R. (2004) Premium principles. Encyclopedia of Actuarial Science, John Wiley \& Sons, Chichester.

MAREK KALUSZKA

Institute of Mathematics

Technical University of Lodz

UL. Zwirki 36

90-924 Lodz - Poland

E-mail: kaluszka@p.lodz.pl 